congenital heart disease there were almost equal numbers in each year up to 7, three-fifths of this number in each year from 8 to 11 , and one-fifth of this number in each year from 12 to 22 , after which there were only scattered cases. The figures suggest that those surviving infancy have no great 'risks until they reach the age of 7 , though clinical experience hardly bears this out. After 7 the chances of surviving become less, and increasingly so after the age of 12 .

If these figures can be taken as giving a true crosssection they mean that only one patient in ten reaches the age of 24 and only two in ten the age of 12. Many parents have been told that their children will not live to 7 or to 14 or to 21 , and there seems some rough truth in these three round numbers as representing periods of increasing danger beyond which survival becomes less likely. During the last few months there have been some deaths after operation, but there have been many more among the other patients I have seen. These considerations seem ample justification for advising operation in spite of the relatively high mortality. The incapacity of many of these cases is hardly realized. A few may lead a reasonable but very quiet life, but if they are old enough to express their views they are generally the greatest enthusiasts for operation, even when the risks are put before them. Many, however, exist rather than live and are a handicap to their parents as well as to themselves, especially when they begin to realize what other children are able to do.

\section{Operative Treatment}

Following Taussig's suggestion about the importance of overcoming pulmonary stenosis, Blalock's experimental work on animals convinced him that a subclavianpulmonary anastomosis was a feasible method, and in October, 1944, Blalock and Taussig introduced their operation for the relief of the inadequate blood-flow to the lungs in Fallot's tetralogy and related lesions.

The operation is essentially an anastomosis between a systemic artery and the pulmonary artery, most commonly an end-to-side subclavian-pulmonary anastomosis, though under special circumstances the innominate or carotid artery may be used or the anastomosis may be end-to-end. In onequarter of these cases the aortic arch will be right-sided instead of curving back as usual to the left and this will of course change the position of the innominate artery. The operation should be performed on the side opposite to the aortic arch because this gives a greater choice of systemic vessels that can be used and the subclavian artery can generally be turned down to the pulmonary artery with a less acute angle.

In infants the small size of the vessels produces a surgical problem of the greatest difficulty, and the operation is much more dangerous and sometimes less lasting in its good effect because the anastomosis is too small as the child grows larger. For this reason the operation should never be performed before the age of 3 , except as a lifesaving emergency, and generally not till 5 . From 5 to 10 is perhaps the ideal age, but there seem no special difficulties in older children, though the difficulties and risks are greater in those over $20-a$ group that should not trouble our successors, as suitable cases should have been operated on earlier. The direct division of the stenosed pulmonary valve by Brock (1948) has opened up fresh possibilities. The late results of this and the indications for it remain to be worked out.

\section{Results of Operation}

The operation is not of course curative in the same way as in the other two groups because the underlying abnormality remains, but the improvement may be so great that the child appears normal to the parents. The immediate upset caused by the operation is less than might be expected, and intravenous fluid is often not required; when it is used only small amounts are needed. Many have a pleural effusion that often needs aspiration, but generally only once; other complications are not common. Of the first 38 cases operated on at Guy's Hospital 6 died, 7 could not have an anastomosis done or did not benefit much, and the remaining 25 were enormously improved almost at once, and this has been maintained.

The immediate results of the operation are excellent, and the improvement seems to be maintained over the period of two to three years for which it has been possible to follow up Blalock's patients. The colour becomes nearly normal except sometimes for slight cyanosis on a cold day or after vigorous exercise, and the patients' capacity is greatly improved so that they can walk some miles and get about normally all day. The polycythaemia disappears quickly, the clubbing of the fingers more slowly. The arm on the side of the divided subclavian artery gives no trouble.

The heart enlarges a little as might be expected from the increased work, but generally only slightly. Sometimes at the beginning the increase during the first few weeks made me feel that any such progressive increase would soon end in disaster, but $I$ have seen no cases in which the increase has continued after the first month or two, and I understand that in the longer American experience this is a rare occurrence, though relapses in those operated on too young and a few instances of congestive heart failure have been seen.

REFERENCES

Benn, J. (1947). Brit. Heart J., 9, 283.

Blalock, A and Taussig, H. B."(1945). J. Amer. med. Ass., 128, 189. Brock, R. C. (1948). British Medical Journal, 1, 1121.

Crafoord, C., and Nylin, G. (1945). J. Thorac. Surg., 14, 347. Gross, R. E. (1939). Ann. Surg., 110, 321.

Peacock, T. B. (1858). Malformations of the Heart, 1st ed., London. Taussig, H. B. (1947). Congenital Malformations of the Heart, New York.

\section{LARGE-INTESTINE COLIC DUE TO SYMPATHETIC DEPRIVATION}

\section{A NEW CLINICAL SYNDROME}

BY

\section{Sir HENEAGE OGILVIE, K.B.E., M.Ch., F.R.C.S. Surgeon to Guy's Hospital}

The subtitle of this note is intentionally provocative. Two cases are described, presenting essentially similar features. In each case the symptoms so strongly suggested obstruction of the distal colon by a carcinoma that laparotomy was eventually undertaken in spite of normal findings in a barium enema. In each case the operation disclosed a normal colon and extensive and entirely unsuspected malignant disease involving the region of the crura of the diaphragm and the coeliac axis and semilunar ganglia.

Whether such a syndrome has been described before I am unaware, but I have not encountered it apart from these two cases, nor have any of my friends whom I have consulted on the subject, and a search of the journals over the last ten years and of the literature of hypertension has failed to reveal similar observations.

\section{Case 1}

In 1944 a physician aged about 66, on the staff of an E.M.S. hospital, began to lose weight, and complained of increasing constipation. He was found to be diabetic, and was treated with diet and insulin. The diabetes was controlled, but the constipation persisted, and he began to have colicky pains in the hypogastrium. On several occasions waves of peristalsis, 
apparently in the pelvic colon, were seen during attacks of colic. A barium meal, and later a barium enema, failed to show any abnormality. The symptoms increased in severity, and after two months the barium enema was repeated; it revealed a dilated and apparently atonic colon, but no localized deformity or obstruction. The attacks of colic continued and he lost weight progressively, so that in spite of the absence of any positive findings a laparotomy was advised.

At operation free fluid was found in the abdomen. The colon was normal throughout. There was a malignant nodule in the peritoneum behind the umbilicus, and a malignant mass on the left crus of the diaphragm, which might have originated in the adrenal, the tail of the pancreas, or the upper pole of the left kidney. The infiltration spread across the region of the aortic opening, and there were metastases in the liver. The nodule in the peritoneum was excised and sent for section, and later was reported to be typical of a secondary deposit of carcinoma of the pancreas.

The patient died three months after operation. No necropsy was performed.

\section{Case 2}

In February, 1948, a solicitor aged 58, who had been perfectly well all his life except for an operation for appendicitis with general peritonitis at the age of 17 , and attacks of dysentery during the first world war, began to suffer from attacks of generalized colicky abdominal pain, accompanied by constipation and relieved by passing wind. Between the attacks, which came on several times a day, he felt well.

On April 1 he was seen by Dr. Geoffrey Evans, who has kindly placed at my disposal the notes he made at the time. The patient told him that the attack had started suddenly six weeks previously with pain in the abdomen, wind, and constipation, and he attributed it to worry and an injudicious feast of whisky and gooseberries. Up to this time his digestion had been good, and his bowe's had acted regularly without laxatives. After the pain started he took a series of aperients and purgatives, which either failed to act or produced a loose motion only. A provisional diagnosis of intestinal obstruction was made, and the patient was referred to Dr. Norman Henderson for a barium enema. Some early diverticulosis of the left colon was reported, but no filling defect. The differential diagnosis of enterospasm was considered, but was rejected because of the obvious distension of the lower part of the abdomen and because bowel function had been normal up to the onset of the illness. There had been some weight loss (about a stone: $6.35 \mathrm{~kg}$.), but this might have been explained by reduced food intake and disturbed sleep due to the pain.

He returned to his home in the country, but the attacks continued, and one which occurred a week later was so severe that it seemed to indicate a commencing obstruction. $\mathrm{He}$ was admitted at once to a nursing-home for observation, when I saw. him for the first time. He was then having bouts of pain three or four times a day. Each started suddenly, with violent hypogastric colicky pain, which made him writhe about and grip the bedclothes. His pulse rose to 100 , but his temperature was unaltered. He did not vomit. During the attack he was tender in the left iliac fossa, and what appeared to be a distended loop of colon could be felt to the right of the tender spot. The attack lasted from half to one hour. When it was over he was happy ; he had no pain, and his abdomen was soft and free from tenderness.

The differential diagnosis between enterospasm and obstruction was again discussed with Dr. Geoffrey Evans, who remained in charge of the case. The patient was of the highly strung type, in whom enterospasm is to be expected. On the other hand, lower abdominal distension, which is not seen in enterospasm, was an obvious feature, and he had never experienced similar pain previously. Sigmoidoscopy and analysis of the faeces showed no abnormality. No formed stool was passed in spite of a variety of daily enemata, atropine by injection, magnesia and belladonna, and paraffin oil $\frac{1}{2} \mathrm{oz}$. (57 ml.) daily. After a week I wrote to his doctor: "I feel we shall be driven to laparotomy, but $I$ feel still more certain that we shall find nothing visibly or palpably wrong. However, he cannot go home as he is, and we will therefore have to operate if we cannot cure him in some other way." The attacks got worse and more frequent.
At operation ten days after his admission to the home no free fluid was found, and the pelvic colon appeared normal except for a temporary ring of spasm-an unusual sight under general anaesthesia. In the subdiaphragmatic region masses of malignant tissue were encountered. All the upper aortic glands, those round the coeliac axis, and those in the lesser. omentum were large and hard, there were large hard nodules in both lobes of the liver, and the falciform ligament and the peritoneum on each side of it were infiltrated. No indication of the site of the primary tumour could be discovered.

The falciform ligament was removed for section and reported on as follows: "The specimen shows the remains of a gland containing considerable secondary deposits of a carcinoma composed of compact groups of small elongated cells with darkly staining nuclei and some abnormal mitoses and without alveolar arrangement. The general appearance somewhat suggests origin in a bronchial carcinoma, possibly of oat-cell type, but the character is very difficult to define more clearly." Another pathologist reported: "I think this is clearly a secondary deposit in connective tissue of an adenocarcinoma, with a tendency to spheroidal change. It would do extraordinarily well for a bronchial neoplasm." A third pathologist considered that the appearance suggested a tumour arising in the adrenal.

The patient died two weeks later. No necropsy was performed.

\section{Discussion}

The symptoms and signs of colonic obstruction in these two cases, occurring in the absence of any organic disease of the colon itself, can be explained in three ways. (1) That the tumour stimulated the parasympathetic supply to the colon. (2) That the tumour cells produced some substance stimulating peristalsis. (3) That the subdiaphragmatic growth had interrupted the sympathetic supply to the large intestine, so that the parasympathetic innervation, which in the distal colon comes from the second and third sacral nerves, was allowed to act unopposed. Of these three explanations the third alone is probable. Infiltration of a motor-nerve bundle should not produce overaction of the muscles it supplies, nor should any kind of mechanical stimulation of nerve fibres in their courses produce repeated overaction: further, in these instances the neurones of the caudal sympathetic system, which supply the section of the bowel involved, were far removed from any abnormal environment. Tumours secreting parasympatheticomimetic substances are, so far as I know, unknown; in any case, the response in these two patients was a local and not a general parasympathetic one.

That the removal of the sympathetic innervation to the colon should lead to excessive and possibly incoordinated contraction of the muscle coats is not improbable. Bilateral ablation of the lumbar sympathetic chain has in a number of instances led to considerable and permanent diminution in the diameter of the bowel in congenital megacolon. Temporary paralysis of the sympathetic system by a spinal analgesic leads to contraction of that part of the alimentary canal supplied by the vagi. Injection of drugs that act on the neuromuscular junction of the parasympathetic system-prostigmin and acetylcholine-causes contraction, often excessive and painful, of the muscular coats of the small and large intestines.

In the two cases under discussion the malignant infiltration surrounded, and may well have put out of action or even destroyed, the splanchnic nerves, the semilunar ganglia, and the coeliac plexus. The vagi passed through the same invaded area, and -were equally liable to interruption-a fact that fits in with the observation that only that part of the bowel supplied by the caudal parasympathetic system gave clinical evidence of the overaction. There is no unanimity among anatomists about where the watershed between the vagal and the sacral supply lies, but it is usually presumed to correspond roughly to the junction between 
the parts of the colon developed from the midgut and hindgut loops - that is, about the distal fourth of the transverse colon.

The most cogent objection to the view that these two cases represent parasympathetic overaction due to sympathetic deprivation is that in each case the contractions were painful. Pain fibres from the small and large intestines reach the cord, so far as we know, by way of the splanchnic nerves. Bentley and Smithwick (1940) showed that the appreciation of pain in the small intestine is abolished after bilateral splanchnicectomy, and Ray and Neill (1947) showed the same in the large intestine. The operation of splanchnicectomy has not, so far as I know, ever been followed by muscular overaction in the bowel.

We thus have two objections to the explanation of sympathetic deprivation put forward to account for the clinical syndrome here discussed-that surgical interruption of the sympathetic nerves at the same site does not produce this effect, and that it does abolish pain, which in these cases was not abolished.

Three suggestions may be made to account for this discrepancy. First, that the interruption of nerve fibres by malignant infiltration may be selective, picking out the motor fibres before the sensory ones. We know that in pressure lesions (tourniquet paralysis and obstetric paralysis) and traction lesions (brachial plexus injuries) involving nerve trunks motor power may be lost with little or no sensory disturbance. In this particular instance the motor fibres (at any rate, the post-ganglionic ones in the plexus) are non-medullated, while the sensory ones are medullated ; and this chemical and histological difference may be accompanied by a differential resistance to infiltration.

Secondly, that the sensory and motor fibres may not run together in all individuals as they do in the majority. One fact that is appearing as the result of the great increase in operations on the sympathetic system is the astonishing and unpredictable course of the sympathetic pathways, particularly at the thoraco-abdominal junction. Boyd (1948) has shown that it is impossible, by the most complete ablation of all known sympathetic nerves and ganglia, to produce functional denervation of the sweat glands in the $\mathrm{D} / 2$ and $\mathrm{L} 1$ areas.

Thirdly, that painful sensations, blocked in their normal pathways, may find others. The motor cortex orders movements, and when the muscles or nerves that normally subserve those movements are destroyed will of ten accomplish the same movements by some other mechanism. The sensory cortex awaits messages, and if the orthodox routes are out of action will often receive them by underground channels. The pain of angina pectoris ceases after bilateral resection of the first four thoracic ganglia, but it comes back eventually. The pain of hip disease is cured for a time by antero-lateral cord section, but the relief is only temporary.

I should like to express my gratitude to Professor F. H. Bentley, Professor Michael Boyd, Sir Stanford Cade, Dr. Geoffrey Evans, Professor F. A. Knott, Professor G. A. G. Mitchell, Professor A. H. T. Robb-Smith, Sir Charles Symonds, and Mr. A. G. M Weddell (the order is alphabetical), with whom I have discussed these cases.

BIBLIOGRAPHY

Bentley, F. H., and Smithwick, Reginald H. (1940). Lancet, 2, 389. Boyd, A. M. (1948). Proc. R. Soc. Med., 41, 370

Mitchell, G. A. G. (1935). Edinb. med. J., 42, 11

Ray, B. S., and Neill, Charles L. (1947). Ann. Surg., 126, 709.

The " Directory of Psychiatric Out-patient Facilities for Adults and Children in England and Wales" may be obtained for 1s. (11s. a dozen) from the National Association for Mental Health, Maurice Craig House, 39, Queen Anne Street, London, W.1.

\section{SOME CAUSES OF SLIGHT CARDIAC ENLARGEMENT}

\author{
BY
}

\section{RONALD HARTLEY, M.R.C.P.}

Assistant Physician, Miller General Hospital and Dreadnought Hospital for Seamen, London

In routine radiological examination cardiac enlargement without physical signs of valvular disease or hypertension is sometimes seen. Among 2,500 recruits examined this problem arose in 25 men, and an attempt has been made to find the cause of the enlargement in 16 of these who were followed up and re-examined three to six years later. Two further cases seen after the original series (Parkinson and Hartley, 1946) are added.

\section{Method}

Opinions differ as to the best method of determining whether true enlargement is present. Hodges and Eyster (1926), Ungerleider and Gubner (1942), and Sherman and Ducey (1944) prefer exact cardiac mensuration. On the other hand, Bedford and Treadgold (1931) say that screen examination is a more reliable means of recognizing heart disease than is actual measurement by orthodiagram. Comeau and White (1942) find prediction tables the best approach to the problem, but both agree that the important factor in estimating heart size is the impression gained by a reliable and experienced radiologist. Parkinson (1936) thought that cardiometry was too complicated for routine clinical use, and that as sound an opinion could be given from screen examination without measurements.

In the type of case discussed measurements are of little use, as they usually fall between the minimum and maximum limits of normal. Careful radioscopy in the three routine positions by more than one experienced observer is more accurate in arriving at a conclusion, and this was the method here adopted. In every case the second examination was made without reference to the previous report. Table I shows the possible causes of radiological enlargement without physical signs of valvular disease or hypertension.

TABle I.-Showing Possible Causes of Radiological Cardiac Enlargement in the Absence of Physical Signs of Valvular Disease or Hypertension

1. Physiological.-Childhood, athleticism, bradycardia, and pregnancy.

2. Apparent.-Scoliosis, depressed sternum, and raised diaphragm.

3. Familial cardiomegaly and congenital idiopathic hypertrophy. Friedreich's ataxia.

4. Organic heart disease--V.D.H. before the development of murmurs, congenital heart disease, repeated coronary thrombosis, cardiac aneurysm, and antecedent hypertension.

5. Abnormal rhythm.-Complete heart-block, paroxysmal tachycardia with failure, auricular fibrillation, and bundlebranch block.

6. Arteriovenous aneurysms and Paget's disease of bone.

7. Metabolic, endocrine, and blood disease-Anaemia, thyrotoxicosis, myxoedema, and beriberi.

Other rarer causes are anomalous coronary arteries, Fiedler's myocarditis, amyloid disease, Gaucher's disease, Von Gierke's disease, and acromegaly.

Among the 18 cases were examples falling into the first four groups only. Table II shows the final conclusion reached. 